\title{
Comparison of the Effects of Different Adduction Loads on EMG Activities of Selected Shoulder Muscles During Shoulder External Rotation Exercise in Healthy Young People
}

\author{
Cheng Peng, PT, MS • Chang-Hwan Bae, PT, PhD • Eun-Hong Choi, PT, MS ${ }^{2}$ Myoung-Kwon Kim, PT, PhD ${ }^{3 \dagger}$ \\ Department of Rehabilitation Sciences, Graduate School, Daegu University \\ ${ }^{1}$ Bae Chang Hwan Training Center, Samsan-ro, Nam-gu, Ulsan, 37, Republic of Korea \\ ${ }^{2}$ Department of Rehabilitation Sciences, Graduate School, Daegu University \\ ${ }^{3}$ Department of Physical Therapy, College of Rehabilitation Sciences, Daegu University
}

Received: November 3, 2018 / Revised: November 22, 2018 / Accepted: March 6, 2019

(c) 2019 J Korean Soc Phys Med

\section{| Abstract |}

PURPOSE: This study was conducted to investigate effects of shoulder adduction load on rotator cuff muscles, including the infraspinatus, during the external rotation exercise of shoulder.

METHODS: This study investigated 16 healthy university students from Daegu University. Before the study started, all participants understood the content of this study. They signed an informed consent form. Five electrodes for surface electromyography (sEMG) were attached to their infraspinatus, middle deltoid, posterior deltoid, upper trapezius and pectoralis major. The participants then underwent the shoulder external rotation exercise with the shoulder adduction at three loads $(0 \mathrm{mmHg}, 20 \mathrm{mmHg}$ and $40 \mathrm{mmHg}$ ) that were controlled using a stabilizer Pressure Biofeedback $^{\mathrm{TM}}$ device. The surface electrodes recorded the

†Corresponding Author : Myoung-Kwon Kim skybird-98@hanmail.net, http://orcid.org/0000-0002-7251-6108

This is an Open Access article distributed under the terms of the Creative Commons Attribution Non-Commercial License (http://creativecommons.org/licenses/by-nc/3.0) which permits unrestricted non-commercial use, distribution, and reproduction in any medium, provided the original work is properly cited. electromyographic data during the external rotation exercise of shoulder.

RESULTS: The infraspinatus was most activated when the shoulder adduction pressure was $40 \mathrm{mmHg}$ during the external rotation exercise of shoulder. The infraspinatus activation significantly increased when the shoulder adduction pressure intensity increased, while the middle deltoid activation and the posterior deltoid activation significantly decreased $(\mathrm{p}<.05)$.

CONCLUSION: In conclusion, increases in shoulder adduction load intensity during shoulder external rotation exercises can have a positive effect on the infraspinatus, which consists of rotator cuff muscles, with minimal activity in the middle and posterior deltoid.

Key Words: Adduction load, Electromyography, Rotator cuff, Shoulder

\section{Introduction}

Very common shoulder disorder with a point prevalence rate of $7 \%$ to $66.7 \%$ [1], is associated with a substantial functional limitation with an increase similar to age. Shoulder pain, impairment and disability are common due 
to rotator cuff dysfunction [2]. Occurring between athletes of repetitive overhead movement sports, this is the most common musculoskeletal problem [3] and chronic shoulder pain, which accounts for $66 \%$ to $85 \%$ of all shoulder cases [4].

Rotator cuff muscles, which consist of the infraspinatus, supraspinatus, subscapularis and teres minor, have long been recognized as an important part of the normal functioning of the shoulder. These muscles stabilize the glenohumeral joint by providing an inferior [5] and anterior point for the humeral head, therefore shoulder joint moves in the center of the glenoid fossa. Many exercises such as scapular plane abduction can strengthen rotator cuff muscles [6]; however, adduction movement is commonly recommended in the rehabilitation of rotator cuff dysfunctions [7]. Alpert et al. [8] and Yasojima et al. [9] reported increasing muscle activity trends in the middle deltoid and rotator cuff with increasing load during abduction from $0^{\circ}$ to $90^{\circ}$ in scapular plane. In contrast, Wilk et al. [10] found that adduction combined with external rotation can increase contraction of the infraspinatus, supraspinatus and teres minor in their rotational role and provide greater potential for strengthening this part of the rotator cuff. However, when resistance exercise is part of a recovery program, it is necessary to consider the contribution and function of the relevant muscle in various exercise loads. Clinical observations suggest that adduction strengthens the rotator cuff and can increase the depressive role of the rotator cuff on the humerus head without activating the superior translation forces of the deltoid [7]. Moreover, when external rotation occurs with adduction it is believed to increase the contraction of the teres minor, supraspinatus and infraspinatus in their rotational role, providing greater potential for strengthening this part of the rotator cuff [10]. Adduction associated with external rotation movement also reduces activity in middle deltoids [11]. Increasing load to increase the pressure intensity level is commonly used in the shoulder rehabilitation to improve muscle strength and function [8]. Electromyography has been used to identify muscle activation patterns and levels during recovery training. Also it has been recommended that movements producing the low muscle activation level be used early in recovery programs [12].

However, it is still not known if the shoulder adduction pressure intensity level affects the rotator cuff muscles. Indeed, few researchers have measured the activity in compensatory muscles (i.e., posterior deltoid and middle trapezius) during the shoulder adduction exercise. Therefore, this study was conducted to determine if there is an effect on the infraspinatus, middle deltoid, and posterior deltoid muscles, or the upper trapezius and pectoralis major when shoulder adduction load increases during shoulder external rotation exercises.

\section{Methods}

\section{Participants}

This study included 16 healthy university students from Daegu University, Korea (mean age, 23.78 \pm 3.19 years; height, $174.47 \pm 5.35 \mathrm{~cm}$; weight, 70.33 $\pm 7.87 \mathrm{~kg}$ ) (Table 1). The inclusion criteria were as follows: no pain on maximally resisted rotation tests, no history of shoulder pain in the previous two years, demonstrated full normal range of shoulder adduction with normal scapulohumeral rhythm [6], no shoulder surgery in the previous two years. The exclusion criteria were as follows: did not demonstrate

Table. 1 General Characteristics of Subjects ( $n=16)$

\begin{tabular}{cc}
\hline Variable & Mean \pm SD \\
\hline Sex (Male/Female) & $16 / 0$ \\
Age (year) & $20.711 \pm .990$ \\
Height (cm) & $160.892 \pm 5.160$ \\
Weight (kg) & $57.651 \pm 9.500$ \\
VAS (score) & $6.714 \pm 3.540$ \\
NDI (score) & $10.575 \pm 9.140$ \\
\hline
\end{tabular}

$\mathrm{SD}$, standard deviation 
normal scapulohumeral rhythm and normal range of movement, no pain on isometric rotation strength tests.

The sample size for this study was calculated using the $\mathrm{G}^{*}$ Power program 3.1.0 (G power program Version 3.1, Heinrich-Heine-University Dusseldorf, Germany). Based on the data from the pilot study, the estimated sample size required to obtain a power at .09 at a five percent significance level was 15 .

\section{Ethics}

Before the start of this study, the study was explained to all subjects and an informed consent form was signed by them. The ethical committee of Daegu University approved this study.

\section{Study Protocol}

Each participant stood with the scapula retracted. Electrodes for electromyography (EMG) were attached to the infraspinatus, middle deltoid, posterior deltoid, upper trapezius and pectoralis major. The participants isometrically conducted shoulder adduction with external rotation (Fig. 1) at three loads $(0 \mathrm{mmHg}, 20 \mathrm{mmHg}$ and $40 \mathrm{mmHg}$ ) that were controlled by a stabilizer Pressure Bio-feedback $^{\mathrm{TM}}$ device (Chatanooga, TN, USA).

For external rotation exercise at 0 degrees of abduction, the exercise protocol consisted of isometric shoulder external rotation in the standing position [13]. To accomplish this, the shoulder was rotated from 30 degrees of external rotation with a resistance using an elastic band (Thera-Band $\mathbb{R}$ Yellow, The Hygenic Corporation, Ohio, USA). A $60 \mathrm{~cm}$ elastic band was then held by hand and elongated to $150 \%$ of the original length when the exercise was started.

Each shoulder test was performed three times with at least 30 seconds of rest between each repetition. The order of the tests was randomized to avoid systematic effects of fatigue. For randomization, sealed envelopes were prepared in advance and marked inside with $\mathrm{A}, \mathrm{B}$, or $\mathrm{C}$ representing $0 \mathrm{mmHg}, 20 \mathrm{mmHg}$ and $40 \mathrm{mmHg}$, respectively.

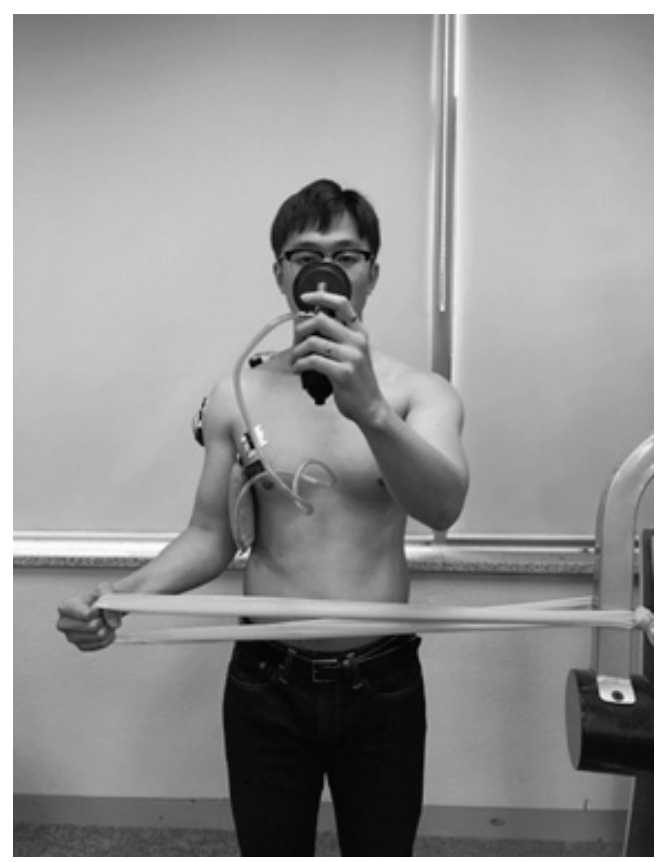

Fig. 1. Experimental posture

This randomization was performed by a third party unaware of the nature of the study.

\section{Surface electromyogram}

Surface EMG data were collected using Desktop DTS (Noraxon USA Inc, Scottsdale, AZ, USA). The sampling rate was $1000 \mathrm{~Hz}$, the raw signal was full-wave rectified and filtered using a Lancosh finite impulse response digital filter (Noraxon USA Inc, Scottsdale, AZ, USA), and the bandpass-filter frequency was between $20 \mathrm{~Hz}$ and $300 \mathrm{~Hz}$. We processed the EMG data into the root mean square (RMS) value, which was calculated from 50-millisecond windows of data points [14]. The electrode sites were shaved and cleaned with rubbing alcohol, after which surface electrode pairs were positioned at an interelectrode distance of $2 \mathrm{~cm}$. We collected the EMG data for the following muscles: infraspinatus $(4 \mathrm{~cm}$ below the spine of the scapula on the lateral aspect over its infrascapular fossa), middle deltoid ( $2 \mathrm{~cm}$ apart on the upper arm, and 
$3 \mathrm{~cm}$ below the acromion), posterior deltoid (lateral border of the spine of the scapula and on an oblique angle toward the upper extremity running parallel to the muscle fiber), upper trapezius (between the cervical spine at C-7 and the acromion) and pectoralis major (2 $\mathrm{cm}$ below the clavicle, just medial to the axillary fold) (Fig. 2)[15]. For normalization, we measured the RMS of a 5-second MVIC three times for each muscle. The first and last second of each MVIC trial was discarded, and the remaining 3 seconds of EMG data were used in the analysis [7]. If a participant was performing an exercise incorrectly, we stopped the exercise and instructed him or her to repeat it. The data for each trial were expressed as a percentage of the calculated mean RMS of the MVIC (\%MVIC), and the mean \%MVIC of the three trials was used in the analysis.

\section{Statistical analysis}

SPSS (IBM Corporation, Armonk, NY, USA) version 22.0 was used for statistical analysis. One-way ANOVA was conducted to compare the activation levels of the five muscles examined across the three loads $(0 \mathrm{mmHg}, 20$ $\mathrm{mmHg}$, and $40 \mathrm{mmHg}$ ). In addition, the LSD test was used to correct for multiple comparisons. Null hypotheses of no difference were rejected if $\mathrm{p}$-values were $<.05$.

\section{Results}

Infraspinatus was most activated during shoulder external rotation when shoulder pressure was highest. Infraspinatus activation significantly increased, while the middle deltoid activation and the posterior deltoid significantly decreased when the shoulder adduction pressure intensity increased during the external rotation exercise of shoulder $(\mathrm{p}<.05)$ (Table 2$)$.

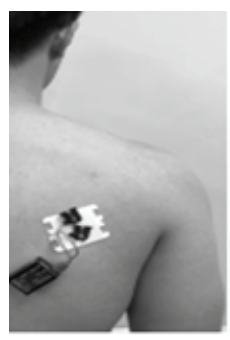

A. Infraspinatus

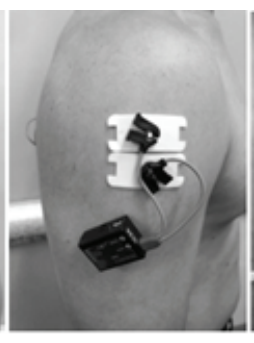

B. Middle deltoid

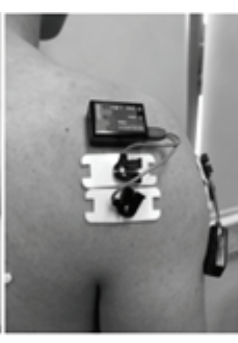

C. Posterior deltoid

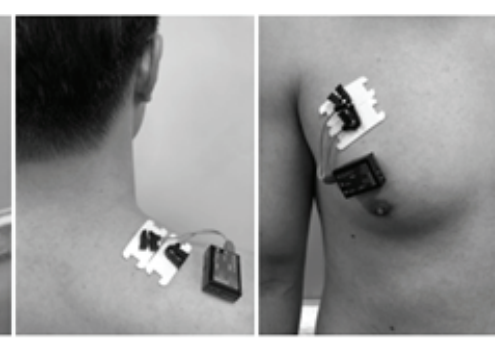

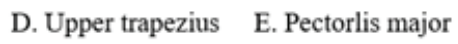

Fig. 2. The position of surface EMG

Table. 2. EMG Activation of Infraspinatus, Middle Deltoid, Posterior Deltoid, Upper Trapezius, Pectoralis Major According to Three Shoulder Adduction Pressure Intensities $(n=16)$

\begin{tabular}{ccccccc}
\hline & $0 \mathrm{mmHg}$ & $20 \mathrm{mmHg}$ & $40 \mathrm{mmHg}$ & $\mathrm{F}$ & $\mathrm{p}$ \\
\hline Infraspinatus & $33.862 \pm 14.533^{\mathrm{a}}$ & $37.392 \pm 15.841$ & $40.362 \pm 17.564$ & 25.223 & $.005^{*}$ \\
Middle deltoid & $32.642 \pm 20.015$ & $29.442 \pm 18.622$ & $25.352 \pm 16.493$ & 8.911 & $.004^{*}$ \\
Posterior deltoid & $35.541 \pm 26.252$ & $32.422 \pm 24.543$ & $27.193 \pm 16.932$ & 5.892 & $.011^{*}$ \\
Upper trapezius & $44.161 \pm 29.321$ & $43.333 \pm 28.734$ & $40.941 \pm 20.341$ & .293 & .752 \\
Pectoralis major & $43.462 \pm 32.601$ & $43.263 \pm 31.462$ & $43.062 \pm 29.394$ & .112 & .901 \\
\hline
\end{tabular}

Mean $\pm \mathrm{SD}^{\mathrm{a},},{ }^{*} \mathrm{p}<.05$, Unit : \%MVIC 


\section{Discussion}

The present trend in the training program is to focus on functional reinstatement by strengthening the specific target muscle with minimum participation of surrounding muscles [16]. Traditional physical therapy programs often use the shoulder exercise to contract the infraspinatus maximally, but several protocols may also result in high levels of the deltoid contraction [7]. The adduction strategy with external rotation has been a critical component of variety training programs to except deltoid activity, therefore retrain the infraspinatus more effectively. In this study, EMG was measured in the infraspinatus, middle and posterior deltoid, upper trapezius and pectoralis major to compare the muscle activity during external rotation according to differences in adduction pressure.

There are several possible explanations for our results. As a part of the transverse force couple, the infraspinatus fulfills a main stabilizing role around the shoulder rather than a principle torque producing role [17]. In contrast, the mechanical quality of the middle deltoid plus posterior deltoid compared with the infraspinatus maintains their having a main torque producing role rather than a stabilizing role [18], similar to the upper trapezius and pectoralis major muscles. Therefore, the load at which adduction trainings are executed and the addition of adduction at another loads may be significant to optimization of the contribution of the infraspinatus and minimization of the deltoid, trapezius and pectoralis major involvement.

Unlike the effects on the middle deltoid and posterior deltoid, increasing adduction pressure did not inhibit the upper trapezius and pectoralis major as anticipated. The upper trapezius is a very weak muscle in the scapular rotation plane, but less well defined in other directions, while the pectoralis major can be targeted from many exercises angles along the clavicle and sternum. Exercises that include horizontal adduction and elbow extension such as the dumbbell, barbell and machine bench press produce high activation of the pectoralis major in the sternocostal part [19]. It is possible that the upper trapezius and pectoralis major were acting as a rotator in the testing position.

It should be noted that this study has some limitations that must be considered when the result is interpreted. First, a small sample size was used because this was an exploratory study. Second, the results of our study are not generalizable because healthy students participated in the study. Therefore, further research is needed to confirm that this result applies to participants with the shoulder pain. Third, we did not measure the activity of other rotator cuff muscles such as the teres minor, which plays a major role as an adductor and external rotator, because of the limited ability of surface EMG to measure the activity of the muscle. Further research using wire EMG is needed to conclude the role of the teres minor during the shoulder external rotation training. Fourth, we did not regulate the speed of movement; however, it affected muscle activity in an EMG study. Thus, further study is needed to measure the muscle activity throughout the ROM during adduction trainings of shoulder with velocity regulated.

\section{Conclusion}

Many people experience a case in which the deltoid, trapezius and pectoralis muscle are first mobilized after the shoulder injury and the rehabilitation exercise is not performed. Thus, in this study, shoulder rehabilitation exercise, which is shoulder stabilizer, was needed.

In conclusion, increases in shoulder adduction pressure intensity during shoulder external rotation exercises can have a positive effect on the infraspinatus with minimum activity in the posterior and middle deltoid.

\section{Acknowledgment}

This material is based on work supported by the Ministry 
of Trade, Industry \& Energy (MOTIE, Korea) under Industrial Technology Innovation Program. No.20001123, 'Advanced design development of design of integrated sleep optimization platform service through core stretching and motion air cell mattress based on pressure sensors and IOT technology.'

\section{References}

[1] Luime J, Koes B, Hendriksen I, et al. Prevalence and incidence of shoulder pain in the general population; a systematic review. Scandinavian Journal of Rheumatology. 2004;33(2):73-81.

[2] Murrell GAC, Hayes K, Walton JR, et al. Reliability of five methods for assessing shoulder range of motion Australian Journal of Physiotherapy. 2001;47:289-94.

[3] Seitz AL, McClure PW, Finucane S, et al. Mechanisms of rotator cuff tendinopathy: intrinsic, extrinsic, or both? Clinical Biomechanics. 2011;26(1):1-12.

[4] Tekavec E, Jöud A, Rittner R, et al. Population-based consultation patterns in patients with shoulder pain diagnoses. BMC Musculoskeletal Disorders. 2012;13: $238-45$.

[5] Hurschler C, Wülker N, Mendila M. The effect of negative intraarticular pressure and rotator cuff force on glenohumeral translation during simulated active elevation. Clinical Biomechanics. 2000;15(5):306-14.

[6] Reed D, Cathers I, Halaki M, et al. Does load influence shoulder muscle recruitment patterns during scapular plane abduction? Journal of Science and Medicine in Sport. 2016;19(9):755-60.

[7] Reinold MM, Wilk KE, Fleisig GS, et al. Electromyographic analysis of the rotator cuff and deltoid musculature during common shoulder external rotation exercises. Journal of Orthopaedic \& Sports Physical Therapy. 2004;34(7):385-95.

[8] Alpert SW, Pink MM, Jobe FW, et al. Electromyographic analysis of deltoid and rotator cuff function under varying loads and speeds. Journal of Shoulder and Elbow Surgery. 2000;9(1):47-58.

[9] Yasojima T, Kizuka T, Noguchi H, et al. Differences in EMG activity in scapular plane abduction under variable arm positions and loading conditions. Medicine and Science in Sports and Exercise. 2008;40(4):716-21.

[10] Wilk KE, Meister K, Andrews JR. Current concepts in the rehabilitation of the overhead throwing athlete The American Journal of Sports Medicine. 2002; 30(1):136-51

[11] Bitter NL, Clisby EF, Jones MA, et al. Relative contributions of infraspinatus and deltoid during external rotation in healthy shoulders. Journal of Shoulder and Elbow Surgery. 2007;16(5):563-8.

[12] Kelly BT, Kadrmas WR, Kirkendall DT, et al. Optimal normalization tests for shoulder muscle activation: an electromyographic study. Journal of Orthopaedic Research. 1996;14(4):647-53.

[13] Malerba JL, Adam ML, Harris BA, et al. Reliability of dynamic and isometric testing of shoulder external and internal rotators. Journal of Orthopaedic \& Sports Physical Therapy. 1993;18(4):543-52.

[14] Ha SM, Kwon OY, Cynn HS, et al. Selective activation of the infraspinatus muscle. Journal of Athletic Training. 2013;48(3):346-52.

[15] Criswell E, Cram JR. Cram's introduction to surface electromyography (2nd). USA. Jones \& Bartlett Publishers. 2011.

[16] Reinold MM, Escamilla R, Wilk KE. Current concepts in the scientific and clinical rationale behind exercises for glenohumeral and scapulothoracic musculature Journal of Orthopaedic \& Sports Physical Therapy. 2009;39(2):105-17.

[17] Sangwan S, Green RA, Taylor NF. Stabilizing characteristics of rotator cuff muscles: a systematic review. Disability and Rehabilitation. 2015;37(12): 1033-43. 
[18] Wattanaprakornkul D, Cathers I, Halaki M, et al. The rotator cuff muscles have a direction specific recruitment pattern during shoulder flexion and extension exercises. Journal of Science and Medicine in Sport. 2011;14(5):376-82.

[19] Schoenfeld B. Science and Development of Muscle Hypertrophy: Human Kinetics. 2016. 